\title{
Comparison of Damage Parameter Based Finite Element Fatigue Life Analysis Results to Combustion Chamber Type TMF Panel Test Results
}

\author{
R. G. Thiede*, E. B. Zametaev ${ }^{\star}$, J. R. Riccius ${ }^{\S}$, \\ DLR Lampoldshausen, Hardthausen, Germany, D-74239 \\ M. Fassin", S. Reese ${ }^{\#}$ \\ RWTH Aachen University, Aachen, Germany, D-52074
}

\begin{abstract}
The inner liner of a regeneratively cooled liquid rocket engine (LRE) combustion chamber is usually exposed to the high temperature of the hot gas and the low temperature of the coolant. It must withstand the pressure within the combustion chamber and the cooling channels. A cyclic operation of a LRE causes a local thinning of the combustion chamber wall (the so called doghouse effect) after a very low number of cycles. Thermomechanical Fatigue (TMF) panels, small actively cooled sections of the hot gas wall of the real engine in combination with cyclic laser heating, are used to study the doghouse effect without the need for testing a full scale engine. An already published numerical Finite Element analysis of the TMF panel on the basis of post-processing Coffin-Manson law resulted in a considerable underestimation of the fatigue life of this TMF panel. In order to improve the prediction of the fatigue life of the TMF panel, a visco-plastic model coupled with isotropic ductile damage was implemented as a user-material in the commercial Finite Element package ANSYS. Furthermore, thermal ageing is implemented in the model in order to take into account the change of the material microstructure with time at elevated temperatures. The temperature dependent material parameters for creep, kinematic and isotropic hardening as well as isotropic damage are determined using data from uniaxial tensile, LCF and stress relaxation tests. The number of cycles to failure is determined numerically and compared to experimental results of the TMF panel. The damage parameter based Finite Element analysis rightly predicts the damage initiation point in the middle cooling channel of the TMF panel and reaches the critical damage (the point where a mesocrack is initiated) at the $122^{\text {nd }}$ cycle instead of the experimentally obtained $174^{\text {th }}$ cycle. The effect of the increase of the wall thickness in the fin areas is also obtained by this numerical analysis. However, this phenomenological approach which does not take into account the crack closure effect is not able to predict the doghouse effect.
\end{abstract}

$\mathbb{1}$
$a, b, c$
$\alpha$
$\alpha_{i}, \beta_{i}, \gamma_{i}$
$b_{i}$
$b_{\text {kin }}$
$C$
$\mathcal{C}$
$D$
$D_{c}$

Nomencla
$=$ identity tensor $\left(2^{\text {nd }}\right.$-order $)$
$=$ thermal ageing parameters
$=$ thermal expansion coefficient
$=$ coefficients for modelling the strain dependen
$=$ isotropic hardening parameters $(i=1,2)$
$=$ kinematic hardening parameter
$=$ kinematic hardening parameter $($ Chaboche $)$
$=$ constitutive elastic tensor $\left(4^{\text {th }}\right.$-order $)$
$=$ isotropic damage
$=$ critical damage parameter

*PhD student, Rocket Engines Department, D-74239 Hardthausen, Germany, non-member

* Senior FE analyst, Rocket Engines Department, D-74239 Hardthausen, Germany, non-member

$\S$ Head of Structures Group, Rocket Engines Department, D-74239 Hardthausen, Germany, senior AIAA member

" PhD student, Institute of Applied Mechanics, RWTH Aachen University, D-52074 Aachen, Germany, non-member

\# Head of Institute of Applied Mechanics, RWTH Aachen University, D-52074 Aachen, Germany, non-member 


\begin{tabular}{|c|c|}
\hline$\Delta \varepsilon_{p}$ & $=$ plastic strain range \\
\hline$\Delta \sigma$ & $=$ stress range \\
\hline$E_{i}$ & $=$ Young's modulus $(i=1,2)$ \\
\hline$\tilde{E}$ & $=$ effective Young's modulus \\
\hline$\varepsilon$ & $=$ strain tensor $\left(2^{\text {nd }}\right.$-order $)$ \\
\hline$\varepsilon_{e}$ & $=$ elastic strain tensor $\left(2^{\text {nd }}\right.$-order $)$ \\
\hline$\varepsilon_{p}$ & $=$ plastic strain tensor $\left(2^{\text {nd }}\right.$-order $)$ \\
\hline$\varepsilon_{p D}$ & $=$ plastic strain at the beginning of damage \\
\hline$\varepsilon_{p R}^{*}$ & $=$ maximum local plastic strain \\
\hline$\varepsilon_{p, e}$ & $=$ plastic-elastic strain tensor $\left(2^{\text {nd }}\right.$-order $)$ \\
\hline$\varepsilon_{p, i e}$ & $=$ plastic-inelastic strain tensor $\left(2^{\text {nd }}\right.$-order $)$ \\
\hline$\varepsilon_{\text {therm }}$ & $=$ thermal strain tensor $\left(2^{\text {nd }}\right.$-order $)$ \\
\hline$\eta$ & $=$ viscosity parameter \\
\hline$\gamma$ & $=$ kinematic hardening parameter (Chaboche) \\
\hline$k$ & $=$ isotropic damage parameter \\
\hline$\dot{\lambda}$ & $=$ plastic multiplier \\
\hline$m$ & $=$ viscosity parameter \\
\hline$N_{R}$ & $=$ number of cycles to rupture \\
\hline$v$ & $=$ direction of plastic flow \\
\hline$v_{i}$ & $=$ Poisson ratio $(i=1,2)$ \\
\hline$p$ & $=$ accumulated plastic strain \\
\hline$p_{D}$ & $=$ accumulated plastic strain damage threshold \\
\hline$\Phi$ & $=$ von Mises yield function \\
\hline$Q_{i}$ & $=$ isotropic hardening parameter $(i=0,1,2)$ \\
\hline$R$ & $=$ drag stress (isotropic hardening) \\
\hline$S$ & $=$ isotropic damage parameter \\
\hline$\sigma_{\text {ageing }}$ & $=$ decrease of the stress due to thermal ageing \\
\hline$\sigma_{\max }$ & $=$ maximum stress of all cycles \\
\hline$\sigma_{\text {ult }}$ & $=$ ultimate stress \\
\hline$\sigma_{\text {vis }}$ & $=$ viscous stress \\
\hline$\sigma_{\mathrm{y}}$ & $=$ yield stress \\
\hline$\sigma$ & $=$ stress tensor $\left(2^{\text {nd }}\right.$-order $)$ \\
\hline$\widetilde{\boldsymbol{\sigma}}$ & $=$ effective stress tensor $\left(2^{\text {nd }}\right.$-order $)$ \\
\hline$T$ & $=$ temperature \\
\hline$t$ & $=$ time \\
\hline $\boldsymbol{X}$ & $=$ back stress tensor $\left(2^{\text {nd }}\right.$-order, kinematic hardening $)$ \\
\hline$X_{o}$ & $=$ initial uniaxial back stress at the beginning of the plastic flow \\
\hline$Y$ & $=$ strain energy release rate \\
\hline$Z$ & $=$ necking factor \\
\hline
\end{tabular}

\section{Introduction}

The development of reusable space vehicles is a demanding task especially for designing reliable and durable rocket engines. The rocket combustion chamber is one of the most critical components of a launcher because it is exposed to extremely high thermo-mechanical loads due to its cyclic operation. The actively cooled inner liner is usually made of copper-base alloys and its behavior is mainly governed by low cycle fatigue and creep at elevated temperatures. A cost-efficient experimental setup with a cut-out model of an actively cooled combustion chamber wall (the so-called TMF-panel) was developed at the German Aerospace Center (DLR) in Lampoldshausen for validating numerical fatigue life prediction analyses [1]. In the present work, a numerical visco-plastic model coupled with ductile isotropic damage based on the work of Tini [2] is used as a user-defined material (USERMAT) in the Finite Element program package ANSYS. Additionally, thermal ageing is implemented in the model in order to describe the decrease of stress during long duration heating. Uniaxial material tests at different temperatures with and without ageing were performed in order to determine the material parameters for creep, isotropic and kinematic hardening, thermal ageing as well as isotropic damage. Finally, the numerically determined number of cycles to failure is compared to experimental data. 


\section{TMF measurements}

\section{A. Temperature measurements}

Previously, nozzle extension type TMF panels made from a nickel basis alloy with a relatively low thermal conductivity were tested at DLR Lampoldshausen [3]. During these tests, the influence of the cooling channel on the temperature distribution can be seen at the first loading cycle already. Now, results of tests with TMF panels that are made from a copper-base alloy are presented, showing a smooth temperature distribution during the early phases of the TMF tests (Figure $1 \mathrm{a}$ )). This smooth temperature distribution is due to the high thermal conductivity of the tested copper-base alloy of $350 \mathrm{~W} /(\mathrm{mK})$. However, the cooling channel structure underneath the thermally loaded structure gets clearly visible shortly before failure (Figure $1 \mathrm{~b}$ ) and c)). Figure $1 \mathrm{~d}$ ) shows the TMF panel with the quarter section and the laser heated region.
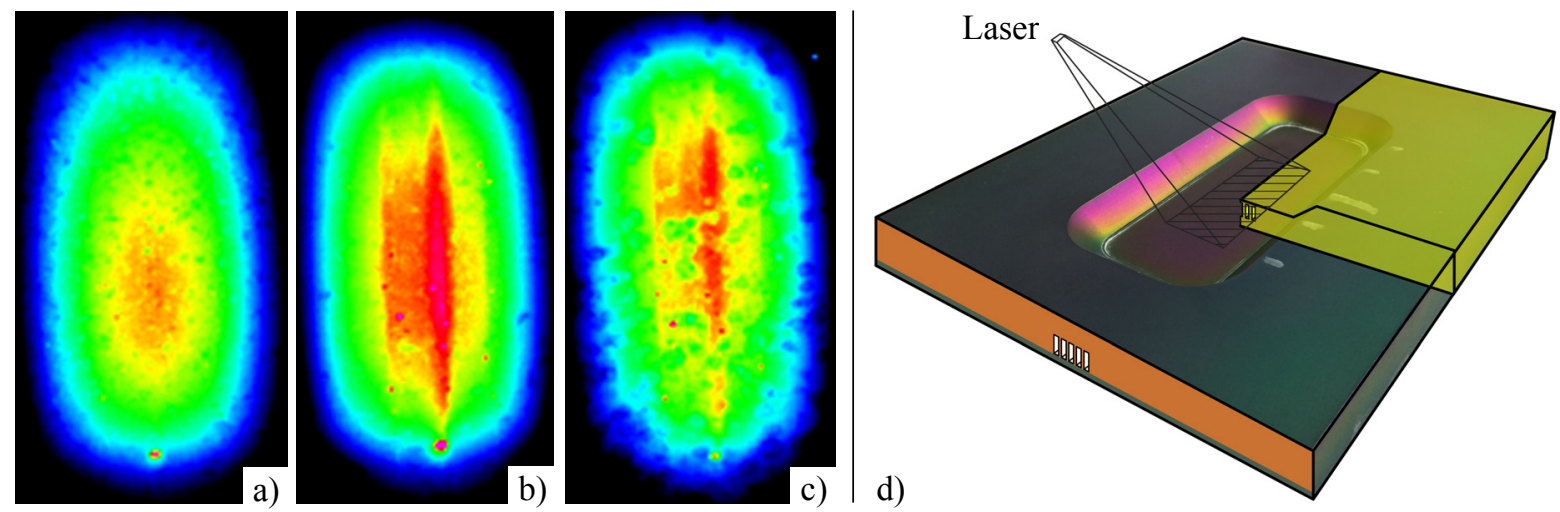

Figure 1: a), b) and c) 2d color plots of the surface temperature of the combustion chamber type TMF panel during different stationary hot runs as measured with an infrared camera; d) illustrated TMF panel with a quarter section highlighted in yellow and laser heated area.

\section{B. Deformation measurements}

The result of the measurement of the deformation during a hot run cycle at about $80 \%$ of the fatigue life of the TMF panel is shown in Figure 2. Dark spots are visible in positons where the image correlation algorithm of the optical deformation measurement system of the TMF test facility has been not able any more to deliver deformation measurement results. This could be caused by either a local loss of the surface coating or a local loss of speckle marks or a strongly reduced contrast between the TMF panel coating and the speckle marks. Red areas indicate the bulging of the fin areas of the TMF panel. This behavior is just the opposite in comparison to previously performed tests with nozzle extension type TMF panels, where the most severe bulging happens in the middle of the cooling channels [3]. The bulging of the fin areas in the generic TMF panel is confirmed by the photographs of the cross section of the TMF panel after cutting it perpendicular to the crack as shown in Figure 3. These photographs also show the crack as well as the thinning of the thermally loaded wall in the vicinity

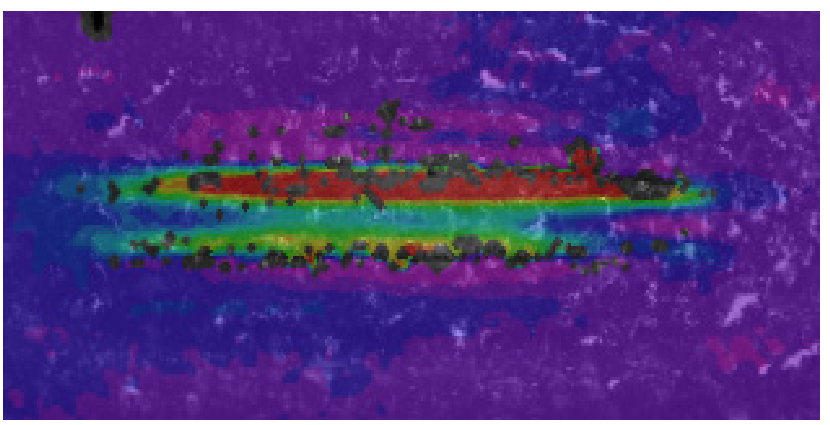

Figure 2: Deformation measured during a hot run of the TMF panel. of the crack.

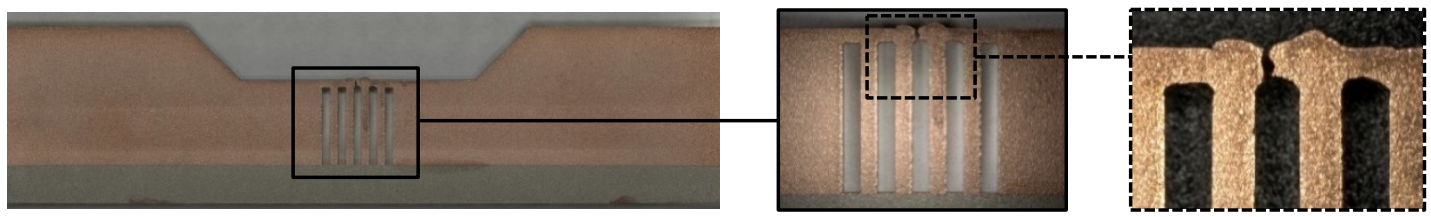

Figure 3: View of a cut-out of the TMF panel with gradually magnified vicinity of the LCF crack. 


\section{Number of cycles to failure}

The crack in the middle cooling channel of the TMF panel as shown in Figure 3 appeared at a total number of 174 cycles. Even taking into account that only the last 93 of the cycles were run at a realistic combustion chamber hot run temperature of $900 \mathrm{~K}$, this is a longer fatigue life than expected. Reasons for the lower number of cycles to failure of real high power core stage combustion chambers like Vulcain are:

- Blanching effects (cyclic oxidation and reduction) of the hot gas surface of the chamber wall [4]

- Localization effect of the chamber wall material deformation due to an injector distance induced temperature variation in circumferential direction of the combustion chamber wall [5]

- Higher heat flux value in radial direction of real combustion chambers - about $80 \mathrm{MW} / \mathrm{m}^{2}$ in the nozzle throat cross section [6] in comparison to the much lower heat flux in thickness direction of the TMF panel of about $20 \mathrm{MW} / \mathrm{m}^{2}$

\section{Finite Element analysis}

\section{A. Finite Element Mesh}

A similar Finite Element mesh as shown in [1] is used. For numerical analysis efficiency reasons, only half of the domain of the TMF panel is modeled. However, in order to account for the influence of the single-sided mounting of the TMF panel (bi metal effect during precooling), the model size was not reduced further to a quarter of the TMF panel. In Figure 4 a), the middle cross section of the meshed TMF panel is shown. 3-D solid elements with quadratic displacement shape functions and full integration have been used. A mesh size of 0.25 $\mathrm{mm}$ in thickness and transversal direction of the TMF panel is used in the middle cross section of the middle cooling channel. A coarse mesh is applied at outer regions and in axial direction of the TMF panel. A symmetry boundary condition is applied at the center area of the TMF panel (on the face normal to the X-direction of the TMF panel).

\section{B. Thermal Finite Element Analysis}

In Figure 4 b), c) and d), the temperature distribution in the hottest cross section of the TMF panel is shown. The thermal expansion at elevated temperatures is taken into account by suitable load steps in the follow-on quasi stationary structural analysis.

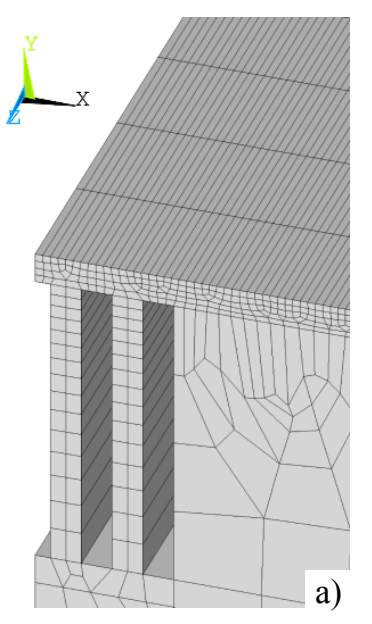

)

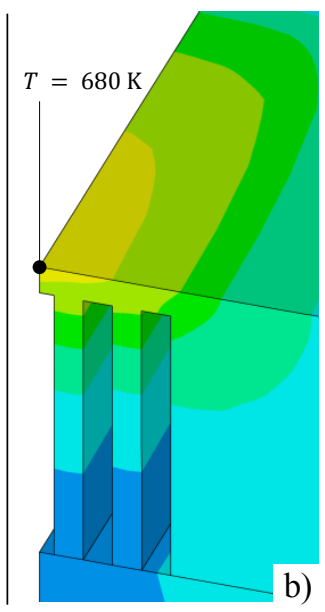

cycle: 1

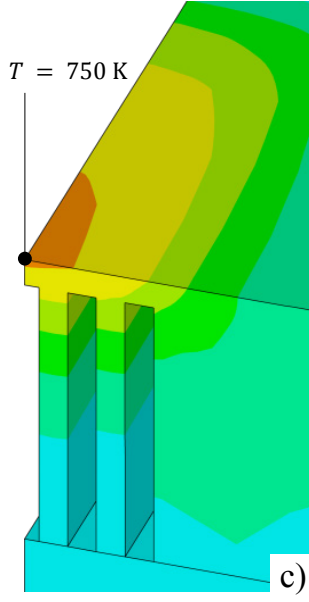

cycles: 2 to 81

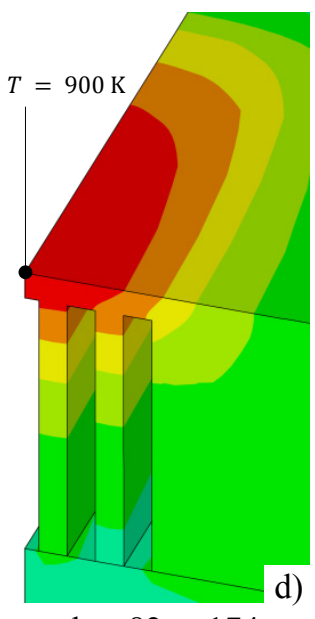

cycles: 82 to 174

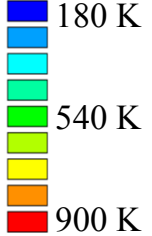

)

$900 \mathrm{~K}$

Figure 4: a) FE-mesh in the middle cross section of the TMF panel; b), c) and d) temperature distributions in the hottest cross section of the TMF panel during the hot run. 


\section{Damage parameter based Finite Element fatigue life analysis}

\section{Basic equations}

A Finite Element integrated visco-plastic model coupled with isotropic damage based on the framework of Tini [2] is used for the fatigue life analysis of the TMF panel. This model is based on the classical rheological model of Armstrong and Frederick [7]. By adding a viscous dashpot $\eta$ (Figure 5), this model is extended into a visco-plastic model. Also, a scalar isotropic damage parameter $D$ is added in order to use the concept of effective stress $\widetilde{\boldsymbol{\sigma}}[8]$ and the principle of strain equivalence [9]

$$
\widetilde{\boldsymbol{\sigma}}=\frac{\boldsymbol{\sigma}}{1-D}
$$

\section{Equation 1}

where $\widetilde{\boldsymbol{\sigma}}=\mathcal{C} \boldsymbol{\varepsilon}_{e}$ is the resulting $2^{\text {nd }}$-order Cauchy stress tensor with $\mathcal{C}$ as the constitutive elastic $4^{\text {th }}$-order tensor. The scalar isotropic damage variable $D$ is defined as

$$
D=1-\left(\frac{\widetilde{E}}{E}\right)
$$

Equation 2

where $E$ is the initial Young's modulus and $\tilde{E}$ is the degraded effective Young's modulus. $\tilde{E}$ represents a loss of stiffness due to damage. The elastic strain tensor $\boldsymbol{\varepsilon}_{e}$ is defined as

$$
\varepsilon_{e}=\varepsilon-\varepsilon_{p}-\varepsilon_{\text {therm }}
$$

Equation 3

where $\boldsymbol{\varepsilon}$ is the total strain tensor, $\boldsymbol{\varepsilon}_{p}=\boldsymbol{\varepsilon}_{p, e}+\boldsymbol{\varepsilon}_{p, i e}$ is the plastic strain tensor decomposed into its elastic and inelastic components. $\boldsymbol{\varepsilon}_{\text {therm }}=\alpha \Delta T \mathbb{1}$ is the thermal strain tensor with the thermal expansion coefficient $\alpha$, the temperature difference $\Delta T$ and the identity tensor $\mathbb{1}$.

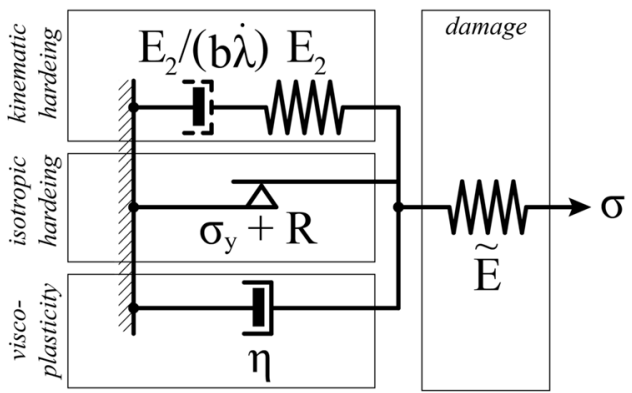

$\widetilde{E} \quad$ effective Young's modulus $\tilde{E}=E(1-D)$

$\sigma_{\mathrm{y}} \quad$ initial yield stress

$R \quad$ drag stress

$E_{2} \quad$ kinematic hardening material parameter

$\dot{\lambda} \quad$ plastic multiplier

$b_{\text {kin }} \quad$ kinematic hardening material parameter

$\eta \quad$ viscosity material parameter

Figure 5: Extension of the elasto-plastic kinematic hardening model according to Armstrong and Frederick into a rate-dependent visco-plastic fatigue life model by adding both the viscous dashpot $\eta$ and isotropic damage variable $D$ [7], [8].

Kinematic hardening was originally described by Armstrong and Frederick [7] and later modified by Chaboche [10]. In the present work, the $2^{\text {nd }}$-order back stress tensor $\boldsymbol{X}$ is defined as

$$
\dot{X}=\frac{2}{3} C \dot{\varepsilon}_{p}-\dot{p} \gamma \boldsymbol{X}
$$

Equation 4

with the scalar back stress $X$ in the uniaxial case

$$
X=v \frac{C}{\gamma}+\left(X_{0}-v \frac{C}{\gamma}\right) e^{-v \gamma\left(\varepsilon_{p}-\varepsilon_{p 0}\right)}
$$

where $C$ and $\gamma$ are the material parameters for kinematic hardening, $\dot{\boldsymbol{\varepsilon}}_{p}$ is the plastic strain rate, $\dot{p}$ is the rate of the accumulated plastic strain, $\boldsymbol{\varepsilon}_{p}$ is the plastic strain and $v=\operatorname{sgn}(\sigma-X)= \pm 1$ corresponds to the direction of the visco-plastic flow. $X_{0}$ and $\varepsilon_{p 0}$ are the initial values of $X$ and $\varepsilon_{p}$ at the beginning of the plastic flow. 
The links between the kinematic hardening parameters of Chaboche and Armstrong and Frederick (Figure 5) which are used in this work are

$$
E_{2}=\frac{2}{3} C\left(1+v_{2}\right) \quad \text { and } \quad b_{\mathrm{kin}}=\sqrt{\frac{2}{3}} \gamma
$$

Equation 6

where $v_{2}=\varepsilon_{x} / \varepsilon_{y}$ is the Poisson ratio in the plastic range. If we assume $v_{2}=0.5$ in case of plasticity, then $E_{2}=C$.

Isotropic hardening (or softening) results in an evolution of the stress range $\Delta \sigma$ with the number of cycles. Voce hardening is used in this model for computing the drag stress $R$ [11]

$$
R=R_{0}+R_{1}+R_{2}=Q_{0} p+Q_{1}\left(1-e^{-b_{1} p}\right)+Q_{2}\left(1-e^{-b_{2} p}\right)
$$

where $R_{1}$ and $R_{2}$ are characterizing the transient slope for small values of the accumulated plastic strain $p$ with the material parameters $Q_{1,2}$ and $b_{1,2}$. The parameter $R_{0}$ defines the linear hardening (or softening) behavior for large values of $p$ with the material parameter $Q_{0}$.

Isotropic damage is described within the effective stress concept as a scalar variable $D$ which is dependent on the degradation of the Young's modulus $E$ during cyclic loading (Equation 2). The evolution of the isotropic damage according to Equation 8 was suggested by Lemaitre [9]

$$
\dot{D}=\sqrt{\frac{2}{3}} \frac{\dot{\lambda}}{1-D}\left(\frac{Y}{S}\right)^{k}\left\langle p-p_{D}\right\rangle
$$

Equation 8

with the strain energy release rate

$$
Y=\frac{1}{2} \varepsilon_{e}: \mathcal{C}: \varepsilon_{e}
$$

Equation 9

$S$ and $k$ are the damage material parameters, $p_{D}$ is the damage threshold and $\mathcal{C}$ is the constitutive elastic $4^{\text {th }}$-order tensor. The symbol $\langle\cdot\rangle$ defines the Macaulay brackets, i.e. $\langle x\rangle=\left\{\begin{array}{l}0, x<0 \\ x, x \geq 0\end{array}\right.$

Viscosity represents the rate-dependent inelastic behavior of materials e.g. creep or stress relaxation. As mentioned already, a viscous dashpot $\eta$ is added to the model of Armstrong and Frederick which is needed for computing the plastic multiplier $\dot{\lambda}$ using the Perzyna formulation [12]

$$
\dot{\lambda}=\frac{\left\langle\bar{\Phi}^{m}\right\rangle}{\eta}
$$

Equation 10

with the von Mises yield function for visco-plasticity

$$
\Phi=\left\|\widetilde{\boldsymbol{\sigma}}^{D}-\boldsymbol{X}^{D}\right\|-\sqrt{2 / 3}\left(\sigma_{\mathrm{y}}+R\right)=\left|\sigma_{\mathrm{vis}}\right|
$$

Equation 11

and the normalized von Mises yield function

$$
\bar{\Phi}=\frac{\left\|\widetilde{\boldsymbol{\sigma}}^{D}-\boldsymbol{X}^{D}\right\|}{\sqrt{2 / 3}\left(\sigma_{\mathrm{y}}+R\right)}-1
$$

where $m$ and $\eta$ are the viscous material parameters, $\sigma_{\text {vis }}$ is the viscous stress, $(\cdot)^{D}$ denotes the deviatoric part of the $2^{\text {nd }}$-order tensor and $\|\boldsymbol{\sigma}\|=\sqrt{\boldsymbol{\sigma}: \boldsymbol{\sigma}}$ the tensor norm. 
Thermal ageing is decreasing the stress over heating time. This effect is included in this model by the means of a $1 / t$ law with the amplification coefficient $a$, the time shift parameter $b$ and the stress shift parameter $c$. These thermal ageing parameters are analytically fitted in order to obtain perfect coincidence for the time points $t_{\text {ageing }}=$ $0 \mathrm{~h}, 10 \mathrm{~h}$ and $20 \mathrm{~h}$ for a given strain $\varepsilon_{i}$

$$
\sigma_{\text {ageing }}\left(\varepsilon_{i}, t_{\text {ageing }}\right)=\left(\frac{a_{i}}{b_{i}+t_{\text {ageing }}}\right)+c_{i} \quad \text { with } \quad i=\{1 ; 2 ; \ldots ; \mathrm{M}\}, \varepsilon_{1}=\varepsilon_{\mathrm{y}}, \varepsilon_{\mathrm{M}}=5 \% . \quad \text { Equation } 13
$$

Denoting $\sigma_{i, t_{j}}=\sigma\left(\varepsilon_{i}, t_{j}\right)$ with $t_{j}=\{0 \mathrm{~h} ; 10 \mathrm{~h} ; 20 \mathrm{~h}\}$ results in the following equations for the strain dependent amplification coefficient, the time shift coefficient and the stress shift coefficient, respectively

$$
\begin{aligned}
& a_{i}=\frac{t_{20 \mathrm{~h}}\left(\sigma_{i, 10 \mathrm{~h}}-\sigma_{i, 0 \mathrm{~h}}\right)\left(\sigma_{i, 10 \mathrm{~h}}-\sigma_{i, 20 \mathrm{~h}}\right)\left(\sigma_{i, 20 \mathrm{~h}}-\sigma_{i, 0 \mathrm{~h}}\right)}{\left(2 \sigma_{i, 10 \mathrm{~h}}-\sigma_{i, 20 \mathrm{~h}}-\sigma_{i, 0 \mathrm{~h}}\right)^{2}} \\
& b_{i}=\frac{-t_{20 \mathrm{~h}}\left(\sigma_{i, 10 \mathrm{~h}}-\sigma_{i, 0 \mathrm{~h}}\right)}{2 \sigma_{i, 10 \mathrm{~h}}-\sigma_{i, 20 \mathrm{~h}}-\sigma_{i, 0 \mathrm{~h}}} \\
& c_{i}=\frac{\left(\sigma_{i, 20 \mathrm{~h}}-\sigma_{i, 0 \mathrm{~h}}\right)^{2}}{2\left(2 \sigma_{i, 10 \mathrm{~h}}-\sigma_{i, 20 \mathrm{~h}}-\sigma_{i, 0 \mathrm{~h}}\right)}+\frac{\sigma_{i, 20 \mathrm{~h}}}{2}+\frac{\sigma_{i, 0 \mathrm{~h}}}{2}
\end{aligned}
$$

Equation 14

Instead of fitting a parameter set $a_{i}, b_{i}$ and $c_{i}$ for each strain value $\varepsilon_{i}$, the evolutions of the three thermal ageing parameters $a, b$ and $c$ are defined as functions of $\varepsilon$ using a double exponential approach. Therefore, twelve coefficients $\alpha_{1 . .4}, \beta_{1 . .4}$ and $\gamma_{1 . .4}$ have to be determined for defining the strain dependency of each of the parameters $a, b$ and $c$ at each temperature

$$
\begin{aligned}
& a(\varepsilon)=\alpha_{1} e^{\alpha_{2} \varepsilon}+\alpha_{3} e^{\alpha_{4} \varepsilon} \\
& b(\varepsilon)=\beta_{1} e^{\beta_{2} \varepsilon}+\beta_{3} e^{\beta_{4} \varepsilon} \\
& c(\varepsilon)=\gamma_{1} e^{\gamma_{2} \varepsilon}+\gamma_{3} e^{\gamma_{4} \varepsilon}
\end{aligned}
$$

Equation 15

Equation 16

Equation 17

The resulting stress tensor $\boldsymbol{\sigma}_{\mathrm{FEM}}$ is computed by subtracting the time dependent thermal stress $\sigma_{\text {ageing }}$ from the stress tensor $\boldsymbol{\sigma}=\widetilde{\boldsymbol{\sigma}}(1-D)$, assuming isotropic damage

$$
\boldsymbol{\sigma}_{\mathrm{FEM}}=\boldsymbol{\sigma}-\left[\begin{array}{ccc}
\sigma_{\text {ageing, } x} & 0 & 0 \\
0 & \sigma_{\text {ageing, } y} & 0 \\
0 & 0 & \sigma_{\text {ageing }, z}
\end{array}\right]
$$

Equation 18

As indicated already in Equation 13, ageing is not taken into account in the case of elasticity, i.e. $\sigma_{\text {ageing }} \leq \sigma_{\mathrm{y}}$.

\section{Material parameter determination}

The tested material $\mathrm{CuCrZr}$ is a cost-efficient copper-base alloy with good thermal conductivity which can be applied in rocket combustion chambers. Uniaxial tests were performed to obtain the material parameters for thermal ageing, creep, kinematic and isotropic hardening as well as isotropic damage.

The experimental test matrix is composed of:

- Tensile tests at $T=\{700 \mathrm{~K} ; 900 \mathrm{~K}\}, \dot{\varepsilon}=0.0021 / \mathrm{s}, t_{\text {ageing }}=\{0 \mathrm{~h} ; 10 \mathrm{~h} ; 20 \mathrm{~h}\}$

- LCF tests at $T=\{300 \mathrm{~K} ; 500 \mathrm{~K} ; 700 \mathrm{~K} ; 900 \mathrm{~K}\}, \dot{\varepsilon}=0.0021 / \mathrm{s}, \varepsilon= \pm 1 \%$

- Stress relaxation tests at $T=\{700 \mathrm{~K} ; 900 \mathrm{~K}\}, t_{\mathrm{hold}}=600 \mathrm{~s}, \varepsilon=\{1 \% ; 2 \% ; \ldots ; 6 \%\}$ 
The material parameters are fitted by either the Levenberg-Marquard method for a nonlinear least square fit with bisquare weights which is obtained with the Matlab Optimization Toolbox ${ }^{\mathrm{TM}}$, or the method of the Conjugated Gradients within ANSYS. Both methods minimize the objective function $f(x)$

$$
\min _{x \in \mathfrak{R}}\|f(x)\|_{2}^{2}=\sum_{i=1}^{N}\left(f(x)_{i, \exp }-f(x)_{i, \text { num }}\right)^{2} \rightarrow \min
$$

The thermal ageing parameters are determined from tensile tests with specimens which were heated in an oven for different periods $t_{\text {ageing }}=0 \mathrm{~h}, 10 \mathrm{~h}$ and $20 \mathrm{~h}$ at elevated temperatures $T=700 \mathrm{~K}$ and $900 \mathrm{~K}$. In Figure 6 , the stress values at these time points as well as the fitted power functions for $900 \mathrm{~K}$ are exemplary shown for strain values of $1 \%$ and $5 \%$.

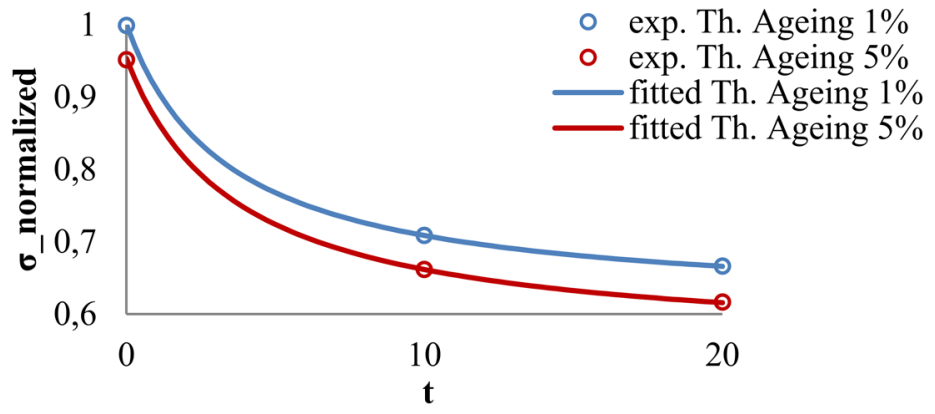

Figure 6: Change of stress over time during tensile tests at $T=900 \mathrm{~K}$.

The power functions determined by the three parameters $a_{i}, b_{i}$ and $c_{i}$ (Figure 6, Equation 13) depend on the strain. As indicated in Equation 13, the strain is determined from values below the limit where the material is strained but still not damaged $(\varepsilon \leq 5 \%$ ). In Figure 7 the strain dependent curves of the three thermal ageing parameters $a, b$ and $c$ are plotted. The experimental data for $T=700 \mathrm{~K}$ show that stress decreases less than $2 \%$ within $20 \mathrm{~h}$ of ageing and therefore it remains within the measurement uncertainty limit. Hence, thermal ageing is not taken into account for the $T=700 \mathrm{~K}$ case. Nevertheless, the parameter $c$ at $T=700 \mathrm{~K}$ is needed for the linear interpolation of the thermal stress e.g. for $T=750 \mathrm{~K}$.
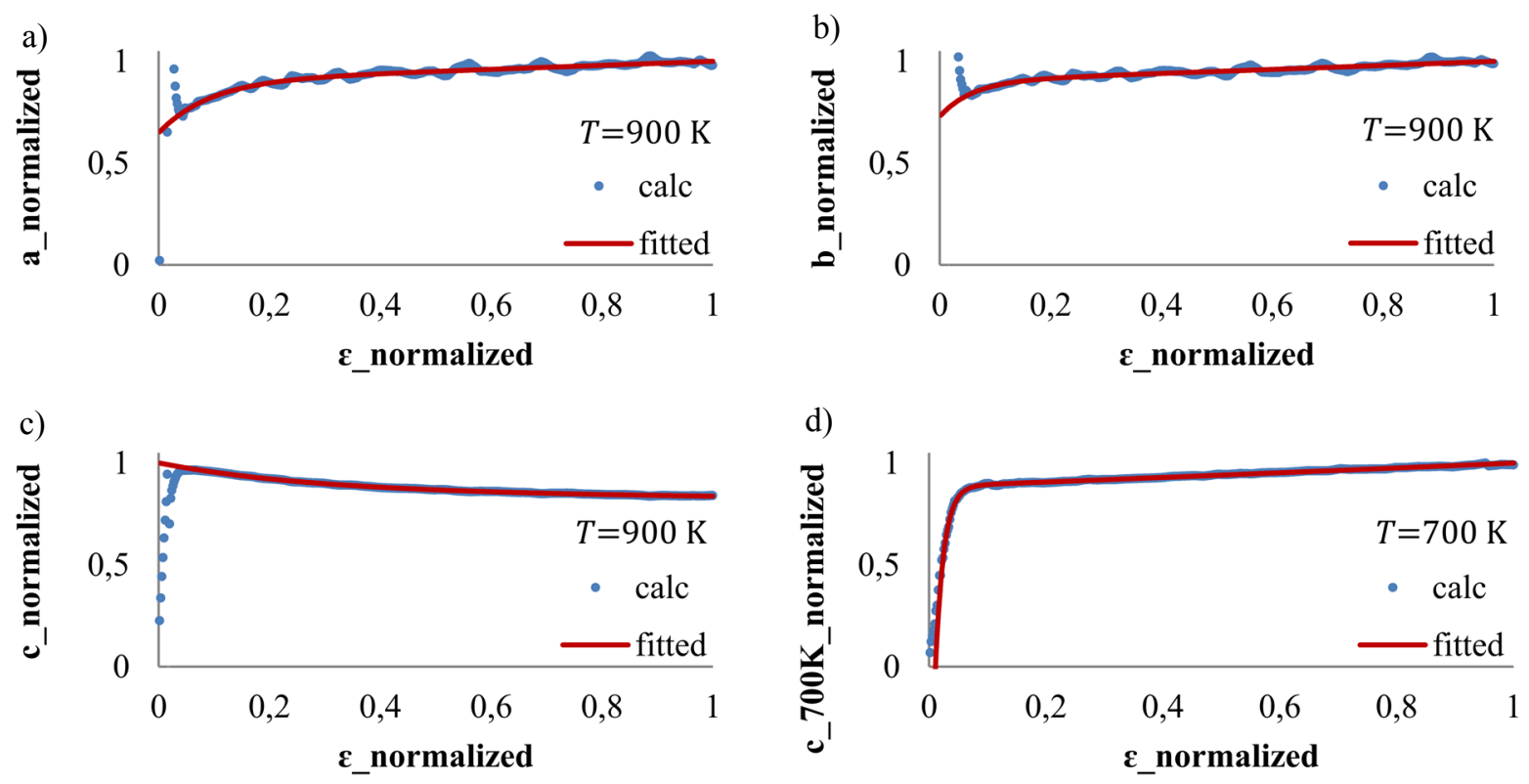

Figure 7: Fitted thermal ageing parameters $a, b$ and $c$ as functions of strain $\varepsilon$ at $T=900 \mathrm{~K}$ and parameter $c$ at $T=700 \mathrm{~K}$. 
The kinematic and isotropic hardening material parameters are identified according to Bouajila [13] who shows a methodology for determining initial values of the hardening parameters for each temperature. Some results of the fitting procedure at $T=900 \mathrm{~K}$ are shown in Figure 8 . The kinematic hardening is fitted for the cycle $N_{R} / 2$, i.e. at half of the number of cycles to rupture at which the isotropic hardening is assumed to have reached saturation. The fitted curves for the isotropic and kinematic hardening show a good agreement with the experimental data as illustrated in Figure 8.

a) - exp. iso. hard. — fitted iso. hard.

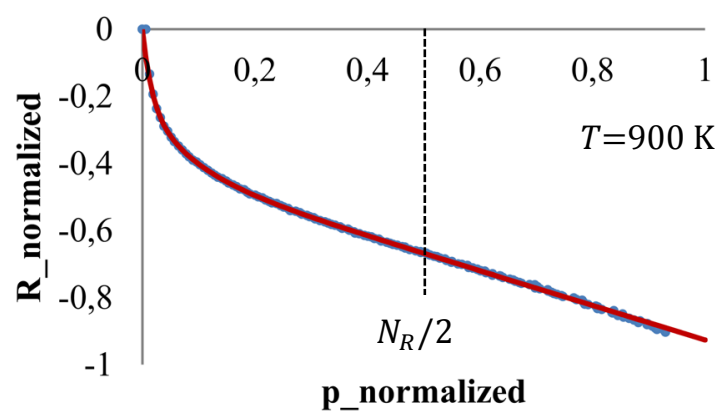

b) exp. kin. hard. fitted kin. hard.

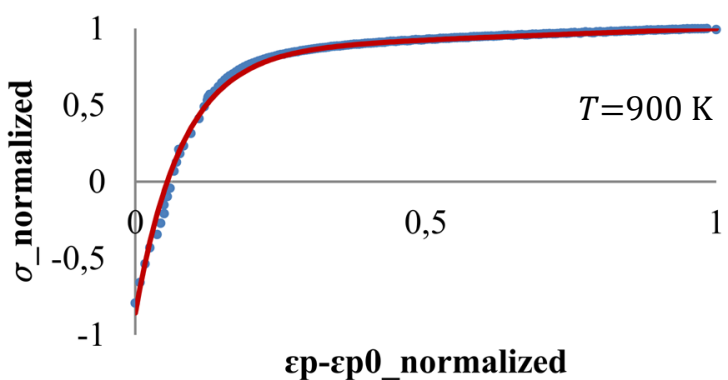

Figure 8: a) Normalized isotropic softening fit at $T=900 \mathrm{~K}$; b) normalized kinematic hardening fit for cycle $N_{R} / 2$ at $T=900 \mathrm{~K}$.

The viscous material parameters are obtained from stress relaxation tests at $T=700 \mathrm{~K}$ and $900 \mathrm{~K}$ with a holding time of $t_{\text {hold }}=600 \mathrm{~s}$. ANSYS is used to model these two uniaxial tests and to optimize the parameters $m$ and $\eta$. Damage could be neglected because the maximum strain of $\varepsilon=6 \%$ is not large enough to lead to a decrease of the Young's modulus during the tests. Figure 9 shows the comparison of the fitted curves and the experimental data for the two temperatures $T=700 \mathrm{~K}$ and $900 \mathrm{~K}$.
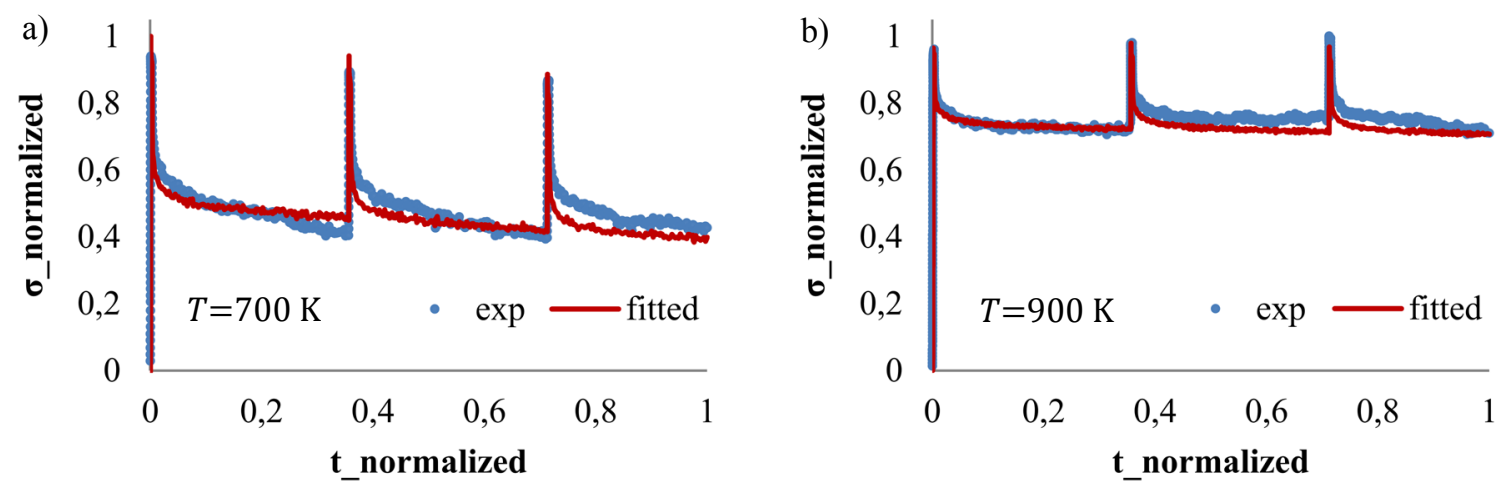

Figure 9: Normalized viscous material behavior which is fitted using the Perzyna formulation.

A quick identification method for the isotropic damage material parameters is described by Lemaitre and Desmorat [14]. The maximum number of cycles $N_{R}$ corresponding to mesocrack initiation $\left(D=D_{c}\right)$ for a uniaxial low cycle fatigue test depends on the unified damage law. The damage parameter $k$ is calculated from Equation 20

$$
N_{R}=\frac{p_{D}}{2 \Delta \varepsilon_{p}}+\frac{1-\left(1-D_{c}\right)^{2 k+1}}{2(2 k+1) D_{c} \Delta \varepsilon_{p}}\left(\frac{\sigma_{\mathrm{ult}}}{\sigma_{\max }}\right)^{2 k}\left(\varepsilon_{p R}^{*}-\varepsilon_{p D}\right)
$$

Equation 20

The remaining parameter $S$ is obtained by solving

$$
D_{c}=\left(\frac{\left(\sigma_{\mathrm{ult}}\right)^{2}}{2 E S}\right)^{k}\left(\varepsilon_{p R}^{*}-\varepsilon_{p D}\right)
$$


where $\Delta \varepsilon_{p}$ is the plastic strain range corresponding to $\sigma_{\max }$ which is the maximum stress value over all cycles (fatigue test). $D_{c}=1-\left(\sigma_{R} / \sigma_{\mathrm{ult}}\right)$ is the critical damage when a mesocrack is initiated with $\sigma_{R}$ as the rupture stress (tensile test). $\sigma_{\mathrm{ult}}$ is the ultimate stress (tensile test), $\varepsilon_{p D} \sim \varepsilon_{p}\left(\sigma_{\mathrm{ult}}\right)$ is the plastic strain where damage starts to occur (tensile test). $\varepsilon_{p R}^{*}=2(1-\sqrt{1-Z})$ is the local plastic strain in the necking area with $Z$ as the necking parameter (tensile test). $p_{D}$ is the damage threshold which can be estimated from the $D$ vs. $p$ - plot (Figure 10).

As shown in Figure 10, the evolution of the isotropic damage had to be determined manually due to the fact that either buckling or a slip of (one of) the extensometer end point(s) occurred during cyclic testing at high temperatures. The damage evolution should be zero until the damage threshold is reached [14]. However, for the considered LCF test case, damage evolves linearly up to the damage threshold $p_{D}$, which limits the linear behavior (Figure 10).

Unexpectedly, the present case showed that the value of $k$ is smaller than zero $(k<0)$ for $T=$ $700 \mathrm{~K}$ and $900 \mathrm{~K}$. A reason for this result could be that the low cycle fatigue (LCF) tests were not performed until the complete rupture of the specimen. Therefore, the Conjugate Gradient optimization method implemented into ANSYS was used to fit the damage parameters $k_{700}$ and $k_{900}$ to the results of tensile tests with a single Element mesh for both temperatures until the critical damage is reached (resulting in the expected $k>0$ for both temperatures).

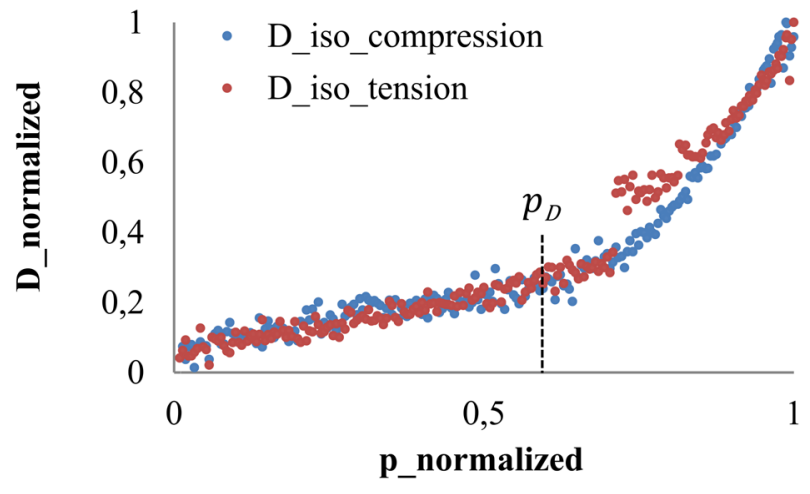

Figure 10: Normalized evolution of isotropic damage caused by degradation of stiffness measured in direction of tension and compression during fatigue testing at $T=900 \mathrm{~K}, \varepsilon= \pm 1 \%, \dot{\varepsilon}=0.0021 / \mathrm{s}$.

\section{Structural Finite Element analysis results}

The structural damage parameter based Finite Element analysis of the TMF panel shows that the critical damage $D_{c}$ is reached during the $41^{\text {st }}$ hot run at $T_{\max }=900 \mathrm{~K}\left(122^{\text {nd }}\right.$ total laser loading cycle).

In Figure 11, the scalar isotropic damage distribution $D$ in the middle cross section of the TMF panel at the numerically predicted rupture cycle 122 is shown. The damage initiation point is located on the coolant side of the middle cooling channel of the TMF panel. The damage distribution throughout the remaining TMF panel is $D=0$, i.e. the accumulated plastic strain has not reached the damage threshold $p<p_{D}$ for any other point (Equation 8).

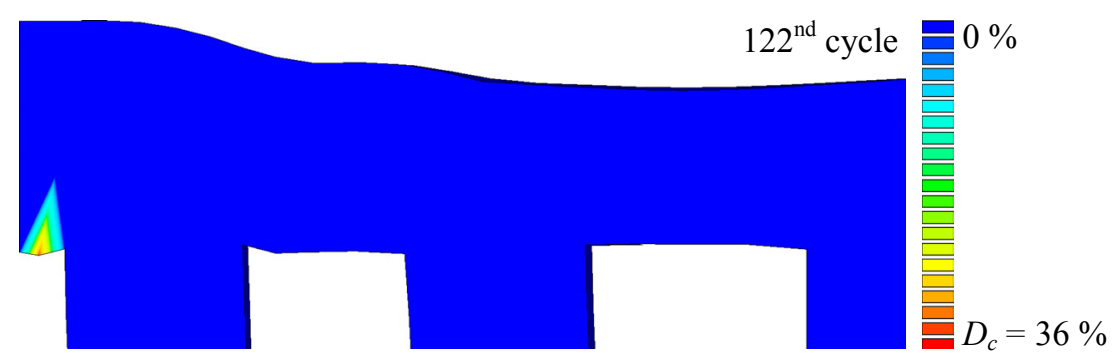

Figure 11: Distribution of the damage $D$ in the middle cross section of the TMF panel at the $122^{\text {nd }} \mathrm{cycle}$

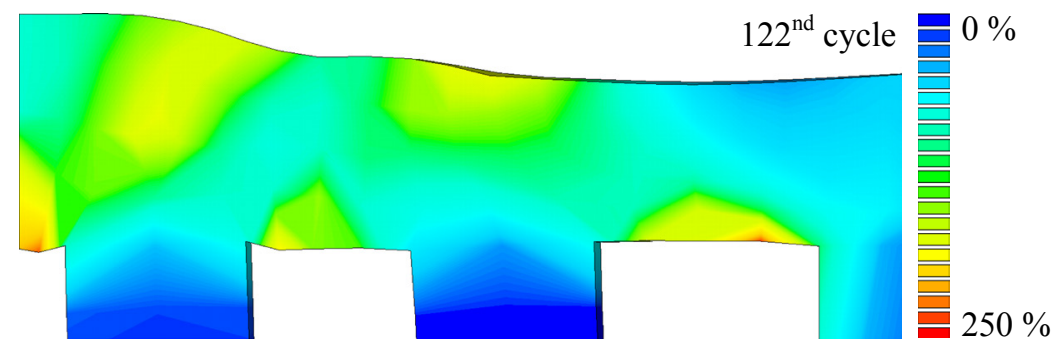

Figure 12: Distribution of the accumulated plastic strain $p$ in the middle cross section of the TMF panel at the $122^{\text {nd }}$ cycle. 
The distribution of the accumulated plastic strain $p$ for this cycle is plotted in Figure 12. The accumulated plastic strain shows two local peaks: one on the coolant side of the middle cooling channel (left) and another one on the coolant side of the outer cooling channel (right). However, according to the color plot shown in Figure 11, damage is predicted to evolve just in the middle cooling channel due to the higher temperature at this position (Figure $4 \mathrm{~d}$ )) and the temperature dependency of the damage threshold $p_{D}$.

\section{Comparison of FE isotropic damage analysis results with experimentally obtained values}

The deformation of the middle cross section of the TMF panel at the experimentally determined rupture cycle (174) and the numerically determined rupture cycle (122) are compared in Figure 13. As the temperature dependency of the damage threshold is taken into account, the simulation results correctly predict the damage initiation point on the coolant side of the middle cooling channel (Figure 11). From the results of the uniaxial LCF tests, a damage threshold relative decrease of $27 \%$ by increasing the temperature from $T=700 \mathrm{~K}$ to $900 \mathrm{~K}$ was determined. Furthermore, the simulated wall thickness increase in the vicinity of the two fins is quite similar to the experimental observations and also similar to the numerical results of Riccius [1].

On the other hand, the numerically predicted number of cycles to failure is $N_{f \text {,FEM }}=122$, which is an underestimation of the experimental result ( 174 cycles) by nearly $30 \%$. Also, the thinning of the middle cooling channel, the so-called doghouse effect, was not obtained by the damage parameter based Finite Element analysis. Furthermore, the decrease of the wall thickness of the outer cooling channel was over-emphasized by the numerical analysis. A possible reason for these discrepancies could be that in the currently considered numerical model with isotropic damage, the crack closure effect is not taken into account. Also, a refinement of the Finite Element mesh would produce a better resolution of the damage distribution as well as a mesh convergency of the results.

Looking just at this first result, it seems that the shown visco-plastic model coupled with isotropic damage can be used for a conservative fatigue life prediction of structures (such as rocket combustion chambers). However, this will have to be confirmed by a larger number of TMF tests with different materials, different temperatures and different hot run (TMF-laser-on) durations. Also, a more ambitious test matrix and improved optimization methods are needed for determining the material parameters considering thermal ageing, creep, isotropic and

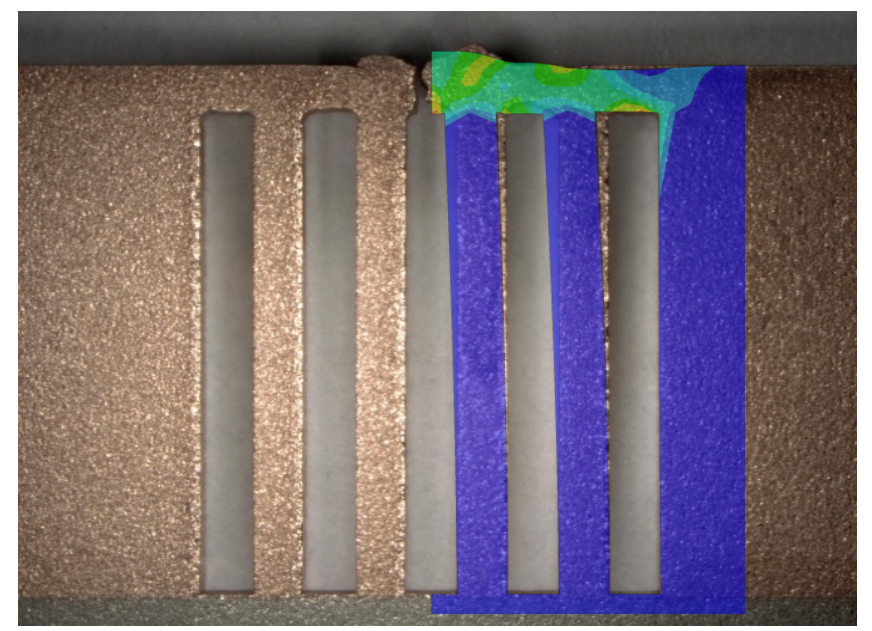

Figure 13: Comparison of deformation between FE analysis results with accumulated plastic strain distribution and experimental results in the middle cross section of the TMF panel. kinematic hardening as well as damage.

\section{Outlook}

In order to improve the agreement between the experimentally obtained and the numerically determined number of cycles to failure, the following numerical improvements will have to be implemented:

- Taking into account the crack-closure effect for acquiring the doghouse effect

- Finer Finite Element mesh for a better resolution and mesh convergency

- Numerical material parameter fitting using a finer mesh size for the uniaxial test specimen

- Taking into account classical creep tests (in addition to the already available stress relaxation tests) for obtaining viscous parameters regarding secondary creep

- Anisotropic damage [15] [16] 


\section{Acknowledgments}

The authors would like to acknowledge:

- Dr.-Ing. Vivian Tini for programming the isotropic damage model source code within the Finite Element Program FEAP

- Dr.-Ing. Wissam Bouajila for his support concerning the definition of the material parameters for creep, isotropic and kinematic hardening

- Dr.-Ing. Andreas Gernoth and Ingo Dörr for the TMF panel test setup and the experimental results

- Scott Beinke for helpful discussions and input

\section{References}

[1] J. R. Riccius, W. Bouajila and E. B. Zametaev, "Comparison of Finite Element analysis and experimental results of a combustion chamber type TMF panel test," in 49th AIAA/ASME/SAE/ASEE Joint Propulsion Conference, San Jose, CA, 2013.

[2] V. Tini and S. Reese, "Lifetime prediction of rocket combustion chamber wall by means of viscoplasticity coupled with ductile isotropic damage," in 11th ASME Biennial Conference on Engineering Design and Analysis (ESDA2012), Nantes, France, 2012.

[3] A. Gernoth, M. Wurdak, J. R. Riccius, S. Schlechtriem, D. Wiedmann, W. Schwarz und L. Brummer, „TMF test based validation of numerical methods for the analysis of heat-loaded walls".AIAA 2010-6887; 46th AIAA/ASME/SAE/ASEE Joint Propulsion Conference \& Exhibit; 25 - 28 July 2010, Nashville, TN.

[4] H. Duval, B. Lefez, T. da Silva Botelho, G. Inglebert, S. Begoc, J. Riccius and B. Hannover, "Experimental investigation of blanching effects of typical rocket combustion chamber wall materials," in 5th European Conference for Aeronautics and Space Sciences (EUCASS), Munich, Germany, 2013.

[5] D. Watanabe, K. Imai, A. Ogawara, K. Kawatsu, H. Negishi, Y. Daimon, N. Tani, N. Yamanishi, H. Sunakawa, A. Kurosu and T. Kobayashi, "Cooling Analysis for Rocket Engine Combustion Chamber with High Fidelity Simulation," in 49th AIAA/ASME/SAE/ASEE Joint Propulsion Conference, San Jose, California, 2013.

[6] D. Preclik, D. Wiedmann, W. Oechslein and J. Kretschmer, "Cryogenic Rocket Calorimeter Chamber Experiments and Heat Transfer Simulations," in 34th AIAA/ASME/SAE/ASEE Joint Propulsion Conference, Cleveland, OH, 1998.

[7] P. J. Armstrong and C. O. Frederick, "A mathematical representation of the multiaxial Bauschinger effect," Central Electricity Generating Board, 1966.

[8] L. M. Kachanov, "Time of the rupture process under creep conditions," Otdelenie tekhnicheskich nauk (in Russian), pp. 26-31, 1958.

[9] J. Lemaitre, A Course on Damage Mechanics, Springer-Verlag, 1992.

[10] J. L. Chaboche, "Constitutive Equations for Cyclic Plasticity and Cyclic Viscoplasticity," International Journal of Plasticity, pp. 247-302, 351989.

[11] E. Voce, "The relationship between stress and strain for homogeneous deformation," Journal of the Institute of Metals, pp. 537-562, 1948.

[12] P. Perzyna, "Fundamental Problems in Viscoplasticity," Advances in Applied Mechanics, pp. 243-377, 1966.

[13] W. Bouajila and J. Riccius, "Identification of the unified Chaboche constitutive model's parameters for a cost efficient copper-based alloy," in Space Proulsion Conference, Cologne, Germany, 2014.

[14] J. Lemaitre and R. Desmorat, Engineering Damage Mechanics: Ductile, Creep, Fatigue and Brittle Failure, Springer-Verlag, 2005.

[15] V. Tini, I. N. Vladimirov and S. Reese, "Influence of anisotropic damage on the lifetime prediction of regeneratively cooled nozzle structure," in Space Propulsion Conference, Bordeaux, France, 2012.

[16] W. Schwarz, D. Wiedmann, S. Schwub, H. Höppel and M. Göken, "Assessment of different continuum damage models for life-time prediction of high-thrust cryogenic combustion chambers," in 4th European Conference for Aerospace Sciences (EUCASS), Moskow, Russia, 2011. 